\title{
Estado nutricional de idosos e prevalência de lesão por pressão na assistência domiciliar ${ }^{*}$
}

\author{
Nutritional status of elderly patients and pressure injury \\ prevalence in home care service
}

Karina Díaz Leyva de Oliveira'• Adriana Haack² • Renata Costa Fortes ${ }^{3}$

\begin{abstract}
RESUMO
O objetivo do estudo foi avaliar o estado nutricional e determinar a prevalência de lesão por pressão (LPP) em idosos assistidos por um serviço de atendimento domiciliar. Trata-se de um estudo transversal descritivo, realizado por meio de visita domiciliar junto à equipe multidisciplinar dos Núcleos Regionais de Atenção Domiciliar Norte e Sul do Distrito Federal. Foram usadas ferramentas de avaliação nutricional como Índice de Massa Corporal (IMC) e Mini Avaliação Nutricional (MAN). Para classificar o estágio das LPP foram considerados os critérios do National Pressure Ulcer Advisory Panel. Amostra formada por 51 idosos, na qual prevaleceu o sexo feminino (76,5\%). A média do IMC no grupo dos idosos sem LPP foi de $24,4 \mathrm{~kg} / \mathrm{m}^{2}$ e nos portadores de lesões, $21,9 \mathrm{~kg} / \mathrm{m}^{2}$. A maioria dos idosos com lesões (54,5\%) apresentou-se desnutrida, segundo a MAN. A prevalência de LPP foi de 21,6\%. A classificação das lesões foi a seguinte: duas (12\%) grau I, cinco (29\%) grau II, sete (41\%) grau III e três (18\%) grau IV. Conclui-se que, segundo a MAN, mais da metade dos idosos com lesão por pressão apresentou desnutrição. A prevalência de LPP entre os idosos foi elevada.
\end{abstract}

Palavras-chave: Idoso; Lesão por Pressão; Prevalência; Avaliação Nutricional; Assistência Domiciliar.

\begin{abstract}
The aim of this study was evaluate the nutritional status and to determine the prevalence of pressure injury (PI) in the elderly assisted by a home care service. This was a descriptive cross study, carried out by means of a home visit to the multidisciplinary team of the Regional Centers for Home Care in the North and South of Federal District. Nutritional tools such as Body Mass Index (BMI) and Mini Nutritional Assessment (MNA) were used. In order to classify the PI stage, the criteria of the National Pressure Ulcer Advisory Panel were considered. Sample formed by 51 elderly people, where the female sex prevailed (76.5\%). The mean BMI in the group of elderly without PI was $24.4 \mathrm{~kg} / \mathrm{m}^{2}$ and of those with injuries, $21.9 \mathrm{~kg} / \mathrm{m}^{2}$. Most of the elderly with lesions (54.5\%) were malnourished, according to MNA. The prevalence of PI was $21.6 \%$. The lesions classification was as follows: two (12\%) grade I, five (29\%) grade II, seven (41\%) grade III and three (18\%) grade IV. In the group of elderly with PI, the mean was classified as moderate risk (14.6). It is concluded that, according to MNA, more than half of the elderly patients with pressure injuries presented malnutrition. The prevalence of pressure ulcer was high.
\end{abstract}

Keywords: Elderly; Pressure Ulcer; Prevalence; Nutritional Assessment; Home Care.

${ }^{1}$ Nutricionista. Programa de Residência em Nutrição Clínica da Secretaria de Estado de Saúde do Distrito Federal. Escola Superior de Ciências da Saúde (ESCS), Brasília-DF. E-mail: karinadloliveira@gmail.com.

Endereço: QI 23, Lt. 08, Ap. 220, Guará II, DF. CEP 71060-230. Autor correspondente.

${ }^{2}$ Nutricionista. Mestrado Profissional em Ciências para a Saúde da Secretaria de Estado de Saúde do Distrito Federal. Escola Superior de Ciências da Saúde (ESCS), Brasília - DF. E-mail: adrianahaack@hotmail.com

Nutricionista. Mestrado Profissional em Ciências para a Saúde da Secretaria de Estado de Saúde do Distrito Federal. Escola Superior de Ciências da Saúde (ESCS), Brasília - DF.E-mail: fortes.rc@gmail.com 


\section{INTRODUÇÃO}

O envelhecimento populacional e o aumento da expectativa de vida ocasionaram modificações no perfil epidemiológico, com predomínio das doenças crônicas não transmissíveis, cujas complicações e sequelas podem resultar em dependência, incapacidades e necessidades de cuidado de longa permanência ${ }^{1}$. Neste contexto, a assistência domiciliar surge como alternativa para o tratamento destes pacientes, com o objetivo de humanizar as práticas de saúde e reduzir custos hospitalares².

Durante 0 envelhecimento ocorrem mudanças fisiológicas que podem afetar o estado nutricional. As alterações no funcionamento digestivo, diminuição do metabolismo, redistribuição da massa corporal, aportes deficitários de calorias e nutrientes, tornam este grupo mais suscetível à desnutrição e deficiências específicas de nutrientes ${ }^{3}$. A desnutrição nos idosos é um problema relevante, relacionado ao aumento das taxas de infecção, transtornos na resposta imune e retardo na cicatrização de feridas ${ }^{4}$.

A idade avançada e as deficiências nutricionais são alguns fatores de risco para o desenvolvimento de lesões por pressão (LPP) ${ }^{5}$, definidas pelo National Pressure Ulcer Advisory Panel (NPUAP) como um dano localizado na pele e/ ou tecido mole subjacente geralmente sobre proeminência óssea, que pode ainda estar relacionado a equipamentos médicos ou outro tipo de dispositivo. Acontece como um resultado de intensa e/ou prolongada pressão ou de pressão combinada com cisalhamento ${ }^{6}$.

$O$ fator idade é considerado um dos indicadores de risco para o desenvolvimento de LPP, principalmente em indivíduos com 65 anos ou mais. A não cicatrização de lesões afeta de três a seis milhões de pessoas com essa idade, e representa $85 \%$ desse evento ${ }^{7-8}$. O desenvolvimento de LPP tem sido observado em $36 \%$ dos idosos com fratura no quadril e de 10 a $35 \%$ no momento da admissão em instituições de longa permanência (ILP) ${ }^{7}$. Em Ribeirão Preto, São Paulo, estudo ${ }^{9}$ que incluía adultos e idosos em assistência domiciliar, encontrou uma prevalência de $21,7 \%$.

Diante do exposto, surgiu o questionamento: qual o estado nutricional e a prevalência de lesão por pressão dos idosos usuários do serviço de atendimento domiciliar?

0 estudo tem como objetivos: avaliar o estado nutricional e determinar a prevalência de lesão por pressão em idosos assistidos por um serviço de atendimento domiciliar da Secretaria de Estado de Saúde do Distrito Federal (SES-DF).

\section{MÉTODO}

Estudo transversal descritivo, realizado por meio de visita domiciliar junto à equipe multidisciplinar dos Núcleos Regionais de Atenção Domiciliar (NRAD) Norte e Sul do Distrito Federal, entre os meses de agosto de 2015 a junho de 2016. Foram incluídos todos os idosos, com idade igual ou superior a 60 anos $^{4}$, cadastrados nos NRADs mencionados, que encontravam-se presentes no dia da visita e concordaram em participar da pesquisa. Foram excluídos pacientes com doenças dermatológicas. A coleta de dados foi realizada pela residente em Nutrição Clínica. O estudo foi aprovado pelo Comitê de Ética em Pesquisas em Seres Humanos da Fundação de Ensino e Pesquisa em Ciências da Saúde, parecer $n^{\circ} 1.167 .815$ e CAAE 47163915.0.0000.5553, atendendo as recomendações da Resolução 466/2012, do Conselho Nacional de Saúde.

Como instrumento de pesquisa foi utilizado um roteiro contendo informações sociodemográficas, de saúde, nutricionais e referentes às LPP. Para avaliação antropométrica foi aferido o peso corporal, a estatura, a circunferência do braço, da panturrilha e a altura do joelho. Para estimativa do peso e da altura de pacientes idosos acamados foram utilizadas as fórmulas propostas por Chumlea et $\mathrm{al}^{10}$. A classificação do estado nutricional, a partir do Índice de Massa Corporal (IMC), foi realizada de acordo com Lipschitz: IMC $<22 \mathrm{~kg} / \mathrm{m}^{2}$ baixo peso, IMC entre 22 e $27 \mathrm{~kg} / \mathrm{m}^{2}$ eutrofia e IMC $>27 \mathrm{~kg} / \mathrm{m}^{2}$ sobrepeso ${ }^{10}$.

O risco nutricional foi avaliado por meio da Mini Avaliação Nutricional (MAN). A MAN compreende dezoito itens agrupados em quatro categorias: avaliação antropométrica; avaliação geral; avaliação dietética e a auto-avaliação sobre problemas nutricionais e estado de saúde. A classificação é feita por meio do somatório de pontos de acordo com cada resposta do questionário, ao final pode-se classificar o paciente em: Normal (12 pontos ou mais na triagem ou $>23,5$ pontos); Desnutrido $(<17$ pontos) e em Risco de desnutrição (17 a 23,5 pontos) $)^{11}$.

Para classificar o estágio das lesões por pressão foram considerados os critérios do NPUAP: estágio ou grau I - eritema da pele intacta que não embranquece após a remoção da pressão; estágio ou grau II - perda da pele envolvendo a epiderme, derme ou ambas, a lesão é superficial e apresenta-se como abrasão ou cratera rasa; estágio ou grau III - perda da pele na sua espessura total, envolvendo danos ou necrose do tecido subcutâneo; estágio ou grau IV - perda da pele na sua total espessura com extensa destruição, necrose dos tecidos ou danos aos músculos, ossos ou estruturas de suporte como tendões ou cápsulas das juntas 5 . A classificação das LPPs foi acompanhada pela equipe de enfermagem.

A avaliação do risco para desenvolver lesão por pressão foi realizada pela Escala de Braden, que permite a análise de aspectos importantes à formação das lesões: percepção sensorial, umidade da pele, atividade, mobilidade, estado nutricional, fricção e cisalhamento. Todos são pontuados de um a quatro, com exceção da fricção e do cisalhamento, cuja pontuação varia de um a três. A pontuação total é estratificada em faixas: <11 pontos, alto risco; 12 a 15 pontos, risco moderado e $>16$ pontos, sem risco ${ }^{12}$.

Os dados obtidos foram armazenados em um banco de dados no Programa Microsoft Excel versão 2010 e sofreram, 
posteriormente, análise estatística descritiva simples com média, desvio padrão e percentual, sendo os resultados obtidos apresentados em tabelas. Na análise estatística foi utilizado o aplicativo SPSS Statistics versão 22.0 (SPSS Inc., (hicago, USA) e utilizados testes como: t-student, Exato de Fisher, Wilcoxon-Mann-Whitney, Spearman e Qui-quadrado. Foram consideradas diferenças significativas valores de $p$ $£ 0,05$.

\section{RESULTADOS}

A amostra foi formada por 51 idosos, dos quais $23,5 \%$ $(n=12)$ eram do sexo masculino e $76,5 \%(n=39)$ do sexo feminino. A média de idade foi de 81,16 \pm 9,71 anos, variando entre 60 e 100 anos. No intervalo de 60 a 69 anos, representaram a amostra $13,7 \%$ idosos, de 70 a 79 anos $19,6 \%$, de 80 a 89 anos $50,9 \%$ e acima de 90 anos $15,8 \%$ (Gráfico 1). Não houve diferença significativa em relação à idade e a presença de $\operatorname{LPP}(p=0,45)$. No grupo que apresentou lesão por pressão, 54,5\% $(n=6)$ eram mulheres e $45,5 \%(n=5)$ homens. A média de idade desse grupo foi de $79,18 \pm 14,04$ anos.

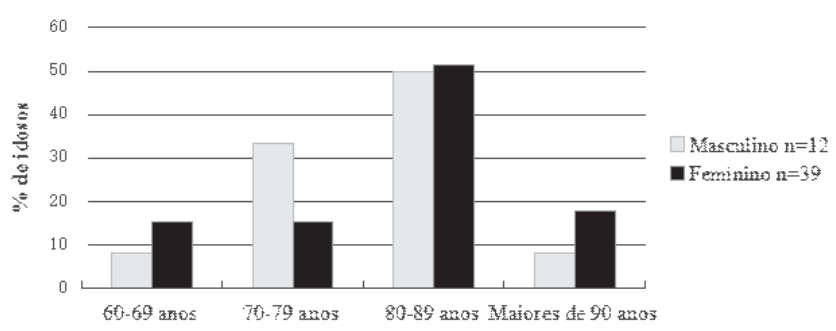

Gráfico 1. Distribuição da idade (\%), segundo faixa etária, dos idosos cadastrados nos Núcleos Regionais de Atenção Domiciliar Norte e Sul do Distrito Federal. Brasília, DF, Brasil, 2015-2016 ( $n=51$ ).

Fonte: dados da pesquisa.

Ao analisar as características sociodemográficas, observou-se que $50 \%(n=20)$ dos idosos sem LPP eram viúvos; $70 \%$ ( $n=28$ ) possuíam cuidadores formais; 47,5\% $(n=19)$ concluíram o ensino fundamental e $65 \% \quad(n=26)$ moravam em casa própria. Em relação aos idosos com LPP, $54,5 \%(n=6)$ eram viúvos; $63,6 \%(n=7)$ possuíam familiares como cuidadores; $45,5 \%(n=5)$ finalizaram o ensino médio e $81,8 \%$ ( $n=9)$ moravam em casa própria (Tabela 1). Não houve associação entre as variáveis e a presença de LPP.

A média do IMC no grupo dos idosos sem LPP foi de $24,4 \pm 4,39 \mathrm{~kg} / \mathrm{m}^{2}$, o valor mínimo foi de 15,4 e o máximo $34,9 \mathrm{~kg} / \mathrm{m}^{2}$. No grupo dos portadores de lesões, o IMC médio foi de $21,9 \pm 4,09 \mathrm{~kg} / \mathrm{m}^{2}$, com valor mínimo de 13,0 e máximo de $27,7 \mathrm{~kg} / \mathrm{m}^{2}$. Foram classificados $29,4 \%(\mathrm{n}=15)$ idosos com baixo peso, 51\% ( $n=26)$ eutróficos e 19,6\% $(n=10)$ com sobrepeso (Gráfico 2). Não houve associação entre a presença de LPP e o IMC $(p=0,349)$.

Ao realizar a Mini Avaliação Nutricional, foram encontrados 19,6\% ( $n=10)$ idosos desnutridos, 52,9\% $(n=27)$ em risco de desnutrição e $27,5 \%(n=14)$ com o estado nutricional adequado (Gráfico 3). A maioria dos

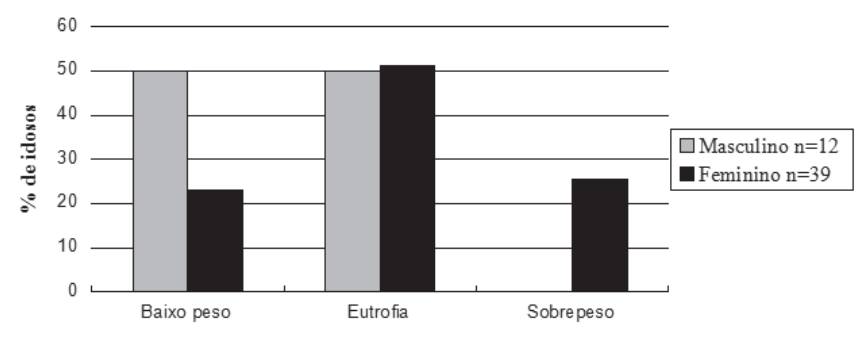

Gráfico 2. Estado nutricional segundo o Índice de Massa Corporal (\%). Brasília, DF, Brasil, 2015-2016 ( $n=51)$.

Fonte: dados da pesquisa.

idosos com lesões apresentou-se desnutrida 54,5\% ( $n=6)$ $27,3 \%(n=3)$ estavam com risco de desnutrição e apenas $18,2 \%(n=2)$ apresentaram o estado nutricional normal. Não houve associação entre a presença de LPP e a desnutrição $(p=0,190)$.

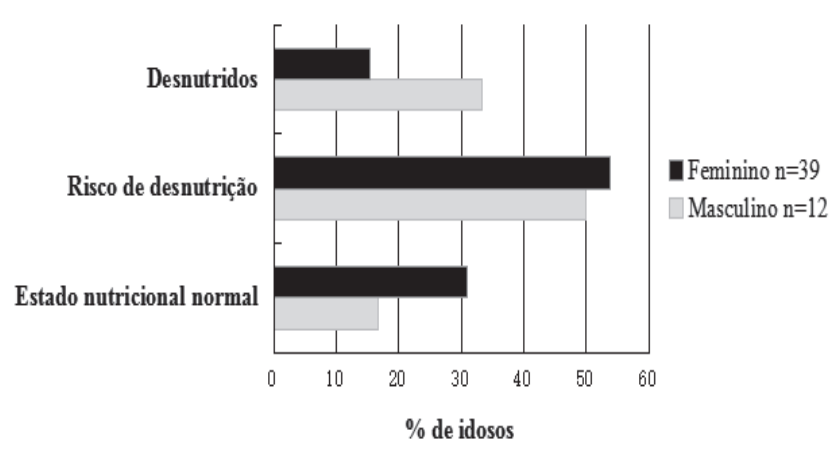

Gráfico 3. Risco nutricional segundo a Mini Avaliação Nutricional (\%). Brasília, DF, Brasil, 2015-2016 ( $\mathrm{n}=51$ ).

Fonte: dados da pesquisa.

A prevalência de LPP foi de 21,6\% $(n=11)$. Dos idosos que apresentavam lesões: $18,2 \%(n=2)$ possuíam duas LPPs; $18,2 \%(n=2)$ três lesões e $63,6 \%(n=7)$ somente uma. Foram encontradas 17 lesões, distribuídas da seguinte maneira: $53 \%(n=9)$ na região sacral; 23,5\% $(n=4)$ nos trocânteres e $23,5 \%(n=4)$ nos calcâneos. Segundo os critérios da NPUAP, foram classificadas $12 \%$ ( $n=2)$ LPP estágio I, 29\% ( $n=5)$ estágio II, 41\% ( $n=7)$ estágio III e estágio IV, 18\% ( $n=3)$. Tinham desenvolvido LPP anteriormente, 38,9\% ( $n=7)$. A média do tempo de lesão foi de 10,8 +7,61 meses.

Em relação à escala de Braden, verificou-se escore médio de 15,5, com variação de 9 a 22 pontos no grupo de idosos sem LPP. No grupo de idosos com lesões a média foi de 14,6, com valor mínimo de 11 e máximo de 23. Dos idosos avaliados, que não apresentaram lesões, 62,5\% $(n=25)$ apresentavam baixo risco para desenvolver LPP; $32,5 \%(n=13)$, risco moderado e $5,0 \%(n=3)$ alto risco. No grupo de idosos com LPP, $27,3 \%(n=3)$ apresentaram baixo risco; $36,4 \%(n=4)$ risco moderado e o mesmo valor, alto risco. A tabela 2 mostra o resultado da aplicação da escala.

Outros fatores de risco encontrados foram: incontinência urinaria em $88,2 \%(n=45)$ dos idosos, imobilização no leito em 74,5\% ( $n=38)$, em 47,1\% ( $n=24)$ doenças crônico degenerativas e em 39,2\% ( $n=20)$ pressão. 
Tabela 1. Características sociodemográficas dos idosos cadastrados nos Núcleos Regionais de Atenção Domiciliar Norte e Sul do Distrito Federal, 2015-2016.

\begin{tabular}{|c|c|c|c|}
\hline \multirow{3}{*}{ Variáveis } & Grupo sem LPP & Grupo com LPP & \multirow{3}{*}{$p$ valor } \\
\hline & $n=40$ & $n=11$ & \\
\hline & n (\%) & $n(\%)$ & \\
\hline Cor da pele & & & 0,684 \\
\hline Branca & $32(80,0 \%)$ & $8(72,7 \%)$ & \\
\hline Parda & $8(20,0 \%)$ & $3(27,3 \%)$ & \\
\hline Estado civil & & & 0,079 \\
\hline Solteiro & $9(22,5 \%)$ & $0(0 \%)$ & \\
\hline Casado & $7(17,5 \%)$ & $4(36,4 \%)$ & \\
\hline Viúvo & $20(50,0 \%)$ & $6(54,5 \%)$ & \\
\hline Divorciado & $2(5,0 \%)$ & $1(9,1 \%)$ & \\
\hline Separado & $2(5,0 \%)$ & $0(0 \%)$ & \\
\hline Tipo de cuidador & & & 0,075 \\
\hline Profissional & $28(70,0 \%)$ & $4(36,4 \%)$ & \\
\hline Familiar & $12(30,0 \%)$ & $7(63,6 \%)$ & \\
\hline Escolaridade de idoso & & & 0,357 \\
\hline Analfabeto & $9(22,5 \%)$ & $1(9,1 \%)$ & \\
\hline Ensino Fundamental & 19 (47,5\%) & $4(36,4 \%)$ & \\
\hline Ensino Médio & $8(20,0 \%)$ & $5(45,4 \%)$ & \\
\hline Ensino Superior & $4(10,0 \%)$ & $1(9,1 \%)$ & \\
\hline Escolaridade do cuidador & & & 0,128 \\
\hline Ensino Fundamental & $11(27,5 \%)$ & $1(9,1 \%)$ & \\
\hline Ensino Médio & $26(65,0 \%)$ & $7(63,6 \%)$ & \\
\hline Ensino Superior & $3(7,5 \%)$ & $3(27,3 \%)$ & \\
\hline Tipo de moradia & & & 0,155 \\
\hline Própria & $26(65,0 \%)$ & $9(81,8 \%)$ & \\
\hline Alugada & $11(27,5 \%)$ & $1(9,1 \%)$ & \\
\hline Cedida (funcional) & $3(7,5 \%)$ & $1(9,1 \%)$ & \\
\hline Renda familiar & & & 0,206 \\
\hline Até 1 salário mínino & $5(12,5 \%)$ & $2(18,2 \%)$ & \\
\hline De 1 a 2 salários mínimos & $7(17,5 \%)$ & $1(9,1 \%)$ & \\
\hline 3 ou mais salários mínimos & $28(70,0 \%)$ & $8(72,7 \%)$ & \\
\hline
\end{tabular}

Fonte: dados da pesquisa.

Tabela 1. Características sociodemográficas dos idosos cadastrados nos Núcleos Regionais de Atenção Domiciliar Norte e Sul do Distrito Federal, 2015-2016.

\begin{tabular}{|c|c|c|c|c|c|c|}
\hline \multirow[t]{2}{*}{ Pontuação } & $\begin{array}{c}\text { Percepção } \\
\text { sensorial }\end{array}$ & Umidade da pele & Atividade & Mobilidade & $\begin{array}{c}\text { Estado } \\
\text { nutricional }\end{array}$ & $\begin{array}{c}\text { Fricção e } \\
\text { cisalhamento }\end{array}$ \\
\hline & n (\%) & n (\%) & n (\%) & n (\%) & n (\%) & n (\%) \\
\hline 1 & $9(17,7 \%)$ & $0(0 \%)$ & $25(49,0 \%)$ & $18(35,2 \%)$ & $0(0 \%)$ & $17(33,3 \%)$ \\
\hline 2 & $10(19,6 \%)$ & $1(2,0 \%)$ & $16(31,4 \%)$ & $21(41,2 \%)$ & $1(2,0 \%)$ & $27(53,0 \%)$ \\
\hline 3 & $15(29,4 \%)$ & $2(3,9 \%)$ & $6(11,8 \%)$ & $6(11,8 \%)$ & $47(92,1 \%)$ & $7(13,7 \%)$ \\
\hline 4 & $17(33,3 \%)$ & $48(94,1 \%)$ & $4(7,8 \%)$ & $6(11,8 \%)$ & $3(5,9 \%)$ & $0(0 \%)$ \\
\hline
\end{tabular}

Fonte: dados da pesquisa.

\section{DISCUSSÃO}

$\mathrm{Na}$ presente pesquisa, houve predomínio do gênero feminino, caracterizando o que a literatura aponta por "feminização da velhice"13. O resultado concorda com o achado de Coelho et al. ${ }^{14}$, que pacientes idosas com idade superior a 80 anos prevalecem no serviço de atendimento domiciliar. A idade elevada é considerada um fator de risco 
para LPP, provavelmente devido às mudanças do processo de envelhecimento da pele. A redução na quantidade $\mathrm{e}$ qualidade de colágeno provoca alterações na elasticidade e turgor da pele. As mudanças na textura da pele atingem a superfície cutânea, fazendo com que ela se apresente fina, lisa ou delgada, demonstrando assim fragilidade ${ }^{15}$.

A maioria dos cuidadores (62,7\%) era profissional contratado. Segundo relatos da família, a aposentadoria do idoso era gasta em medicamentos e com o cuidador formal. Este indivíduo convive diariamente com o idoso, cuida da alimentação, das atividades de reabilitação, da higiene e muitas vezes, da interação com a equipe multidisciplinar ${ }^{16}$. Menos de $30 \%$ das famílias recebiam abaixo de 3 salários mínimos. Este resultado confirma a Pesquisa de Orçamentos Familiares (POF), realizada pelo Instituto Brasileiro de Geografia e Estatística (IBGE) em 2008-2009, na qual a renda familiar do brasiliense era quase o dobro da média nacional ${ }^{17}$.

Existem poucos estudos realizados no Brasil sobre avaliação nutricional em idosos com assistência domiciliar. O percentual de idosos com baixo peso segundo o IMC, nesta pesquisa, foi superior ao estudo ${ }^{18}$ realizado em Instituição de Longa Permanência para Idosos (ILPI) em Porto Alegre-RS (16\%), que teve maior número de idosos com sobrepeso (31,3\%). Em outra ILPI, em FortalezaCE, 22,2\% apresentaram baixo peso, enquanto que a classificação de sobrepeso foi similar $(20,4 \%)^{19}$. Vale ressaltar que somente as idosas apresentaram excesso de peso. Estudo ${ }^{20}$ realizado em Minas de Gerais encontrou que a prevalência de excesso de peso foi maior entre as mulheres $(84,4 \%)$ quando comparada a dos homens $(15,4 \%)$. O IMC é um indicador antropométrico muito usado para avaliar o risco nutricional, por ser facilmente aplicável, de baixo custo e não invasivo. 0 emprego desta ferramenta em idosos apresenta controvérsias, devido à redução do tecido adiposo, da estatura, da massa corporal magra e da quantidade de água no organismo ${ }^{18}$.

0 primeiro instrumento desenvolvido para avaliar o risco nutricional em idosos foi a Mini Avaliação Nutricional, capaz de identificar os que possam se beneficiar de intervenção precoce ${ }^{11}$. Segundo a MAN, $27,5 \%$ de todos os idosos tinham um estado nutricional adequado, resultado inferior aos 61,2\% encontrados em Bogotá, com idosos da comunidade ${ }^{21}$. Moreira et al. ${ }^{22}$ realizaram pesquisa com idosas residentes em instituição geriátrica, 14,3\% eram desnutridas e $78,6 \%$ tinham um estado nutricional adequado. Os valores encontrados na população feminina neste estudo foram $15,4 \%$ e $30,8 \%$ respectivamente.

No estudo, apesar de mais da metade dos portadores de lesão por pressão apresentar desnutrição segundo a MAN, esta não foi associada com a presença de LPP, entretanto é reconhecido que a desnutrição é um importante fator de risco para o desenvolvimento da mesma ${ }^{5}$. A gravidade da desnutrição eleva a probabilidade de ter uma lesão por pressão mais grave ou um maior número de lesões. Uma pesquisa observou que a LPP e sua severidade foram diretamente relacionadas à desnutrição, ou seja, 98\% das pessoas que apresentaram gravidade da LPP superior ou igual ao grau II eram desnutridas ${ }^{23}$. 0 estado nutricional interfere diretamente na reparação tecidual. A desnutrição está relacionada à cicatrização inadequada, pela diminuição da produção de fibroblastos, de neoangiogênese e de síntese de colágeno, além de menor capacidade de remodelação tecidual ${ }^{24}$.

No âmbito nacional existem poucos estudos que investigaram a LPP em idosos no domicílio. Um deles, realizado por Coelho et al. ${ }^{14}$, encontrou uma prevalência de $31,4 \%$ de LPP.Estudo ${ }^{10}$ realizado em domicílios com pacientes adultos e idosos que estiveram hospitalizados e receberam alta da Unidade de Emergência do Hospital das Clínicas da Faculdade de Medicina de Ribeirão Preto, da Universidade de São Paulo, encontrou $21,7 \%$, resultado quase igual ao desta pesquisa. Outro estudo ${ }^{25}$, realizado em pacientes residentes na área de cobertura da Estratégia de Saúde da Família (ESF) de Teresina-PI, na qual a maioria (79,41\%) da amostra era idosa, a prevalência de LPP foi de $23,5 \%$ e a maior parte foi encontrada na região sacral (79,16\%).

Apesar de o estudo ter encontrado percentual elevado de incontinência urinária (88,2\%), ao aplicar a escala de Braden os familiares referiram fazer a troca de fralda com bastante regularidade, com o objetivo de evitar a umidade da pele. Dos pacientes que foram avaliados com pontuação um e dois da escala, os fatores que mais contribuíram para o desenvolvimento de LPP foram: fricção e cisalhamento $(86,2 \%)$, falta de atividade $(80,4 \%)$ e falta de mobilidade (76,5\%). Estudo ${ }^{16}$ realizado no Hospital Universitário da Universidade de São Paulo encontrou o mesmo resultado, com valores de $94,5 \%, 82,4 \%, 76,5 \%$, respectivamente. Pesquisa realizada com idosos internados em Unidade de Terapia Intensiva, em Pernambuco, encontrou que $85 \%$ possuiam problemas em relação à fricção e cisalhamento, $95 \%$ estavam restritos ao leito e $85 \%$ encontravam-se ocasionalmente úmidos ${ }^{26}$.

Este trabalho apresentou como limitação o pequeno tamanho da amostra. A pesquisas encontradas na literatura sobre avaliação nutricional de idosos abrangia também os da comunidade ou residentes em ILPI, não apenas usuários de assistência domiciliar, assim como os estudos de prevalência de lesão por pressão, nos quais foram incluídos adultos também.

\section{CONCLUSÃO}

Mais da metade dos idosos com lesões estavam desnutridos, segundo a MAN, porém não houve associação com a presença de LPP. A avaliação nutricional realizada precocemente constitui uma ferramenta fundamental na identificação de idosos desnutridos ou em risco nutricional, permitindo a instituição da terapia nutricional adequada. Neste contexto é importante o papel do nutricionista, profissional capacitado para realizar intervenções que podem evitar o deterioro da saúde e futuras complicações, 
assim como melhorar o estado nutricional e a qualidade de vida do idoso.

A prevalência de LPP em idosos na assitência domiciliar foi elevada. Tendo como base os principais fatores predisponentes para o desenvolvimento das lesões por pressão e o perfil dos idosos em atendimento domiciliar, o conhecimento da prevalência destas lesões pode contribuir para a melhoraria da assistência prestada e implementação de estratégias de redução destes agravos.

\section{REFERÊNCIAS}

1. Pereira IFS, Spyrides MHC, Andrade LMB. Estado nutricional de idosos no Brasil: uma abordagem multinível. Cad. Saúde Pública. 2016; 32(5): e00178814.

2. Diniz EM, Morita ABPS, Paula MAB. Situação de risco para úlceras por pressão em uma unidade de Assistência Domiciliar. Revista Estima. 2016;14(2):53-60.

3. Freitas AF, Prado MA, Cação JC, Beretta D, Albertini S. Sarcopenia e estado nutricional de idosos: uma revisão da literatura. Arq. Ciênc. Saúde. 2015; 22(1):09-13.

4. López MTF, Baamil OF, Doldán CL, Alonso MLB, Prada MTS, Labrador FL et al. Prevalencia de desnutrición en pacientes ancianos hospitalizados no críticos. Nutrición Hospitalaria. 2015; 31(6):2676-84.

5. Pedroni L, Bonatto S, Mendes K. O impacto da desnutrição no desenvolvimento e na gravidade das úlceras por pressão: uma revisão da literatura. RBCEH. 2014; 11(1):89-102.

6. National Pressure Ulcer Advisory Panel. Pressure Ulcer Stages Revised.Washington. [Internet]. 2016; [acesso em 10 de agosto de 2016]. Disponível em: http://www.npuap.org/about-us/.

7. Blanc G, Meier MJ, Stocco JGD, Roehrs H, Crozeta K, Barbosa DA. Efetividade da terapia nutricional enteral no processo de cicatrização das úlceras por pressão: revisão sistemática. Rev Esc Enferm USP. 2015; 49(1):152-61.

8. Moraes JT, Borges EL, Lisboa CR, Cordeiro DCO, Rosa EG, Rocha NA. Conceito e classificação de lesão por pressão: atualização do National Pressure Ulcer Advisory Panel. Enferm. Cent. O. Min. 2016 mai/ago; 6(2):2292-2306.

9. Moro JV,Caliri MHL.Úlcera por pressão após a alta hospitalar e o cuidado em domicílio. Esc Anna Nery. 2016; 20(3):e20160058.

10. Rosa COB, da Silva BP, Balbino KP, Ribeiro SMR, Ribeiro AQ Firmino $\mathrm{HH}$. Avaliação Nutricional de indivíduos internados em um hospital geral. O Mundo da Saúde. 2014;38(4):430-8.

11. Soares ALG, Mussoi TD. Mini-avaliação nutricional na determinação do risco nutricional e de desnutrição em idosos hospitalizados. Rev Bras Nutr Clin 2014; 29 (2): 105-10.

12. Borghardt AT, Prado TN, Araújo TM, Rogenski NMB, Bringuente MEO. Evaluation of the pressure ulcers risk scales with critically ill patients: a prospective cohort study. Rev. LatinoAm. Enfermagem. 2015; 23(1):28-35.

13. Santos AA, Mansano-Schlosser TCS, Ceolim MF, Pavarini SCI.Sleep, fragility and cognition: a multicenter study with Brazilian elderly. Rev. bras. enferm. 2013; 66(3): 351-7.

14. Coelho ADA, Lopes MVO, Melo RP, Castro ME. O idoso e a úlcera por pressão em serviço de atendimento domiciliar. Rev Rene. 2012; 13(3):639-49.
15. Duarte AVS, Alves MA. Úlcera de pressão: fatores de risco em pacientes hospitalizados. Rev. Diálogos Acad. 2013; 2(2):102-8

16. Pereira RA, Santos EB, Fhon JRS, Marques S, Rodrigues RAP. Sobrecarga dos cuidadores de idosos com acidente vascular cerebral. Rev Esc Enferm USP. 2013; 47(1):185-92.

17. Brasil. Ministério de Planejamento, Orçamento e Gestão. Instituto Brasileiro de Geografia e Estatística - IBGE. Pesquisa de Orçamentos Familiares 2008-2009. Despesas, rendimentos e condições de vida. Rio de Janeiro. 2010.

18. Souza R, Fraga JS, Gottschall CBA, Busnello FM, Rabito El. Avaliação antropométrica em idosos: estimativas de peso e altura e concordância entre classificações de IMC. Rev. Bras. Geriatr. Gerontol. 2013; 16(1):81-90.

19. Borges CL, Silva MJ, Clares JWB, Nogueira JM, Freitas MC. Características sociodemográficas e clínicas de idosos institucionalizados: contribuições para o cuidado de enfermagem. Rev Enferm UERJ. 2015 mai/jun;23(3):381-7.

20. Sá SC, Souza AB, Andrade JMO, Gonçalves JTT, Assis JR, Oliveira MVM. Perfil nutricional de idosos e sua associação com fatores cognitivos e sociodemográficos. Rev Enferm UFPE. 2017; 11(4):1685-91.

21. Santos ALM,Amaral TMSPF, Borges PGFB. Subnutrição e fatores associados em uma comunidade de idosos Portugueses. Rev. Nutr. 2015; 28(3):231-240.

22. Moreira JPD, Melo PTH, Oliveira AMC. Perfil nutricional de idosas residentes em uma instituição geriátrica. Rev Bras Promoç Saúde, Fortaleza. 2012 jan./mar;25(1): 52-8.

23. Brito PA, Generoso SV, Correia MITD. Prevalence of pressure ulcers in hospitals in Brazil and association with nutritional status - A multicenter, cross-sectional study. Nutrition. 2013; 29 (4): 646-49.

24. Sociedade Brasileira de Nutrição Parenteral e Enteral. Associação Brasileira de Nutrologia. Sociedade Brasileira de Clínica Médica. Projeto Diretrizes. Terapia Nutricional para Portadores de Úlceras por Pressão [Internet]. 2011; [acesso em 10 de agosto de 2016]. Disponível em: https://diretrizes. amb.org.br/_BibliotecaAntiga/terapia_nutricional_para_ pacientes_portadores_de_ulceras_por_pressao.pdf.

25. Bezerra SMG, Luz MHBA, Andrade EMLR,Araújo TME, Teles JBM, Caliri MHL. Prevalência, fatores associados e classificação de úlcera por pressão em pacientes com imobilidade prolongada assistidos na Estratégia Saúde da Família. Revista Estima. 2014; 12(2).

26. Sousa Júnior BS, Mendonça AEO, Duarte FHS, Silva CC. Riscos para o desenvolvimento de úlceras por pressão em idosos utilizando as subescalas de Braden. Revista enfermagem atual in derme. [Internet]. 2016 [acesso em 02 out 207]; 77 (15): 37-43. Disponível em: http://inderme.com.br/revistas/ revista15.html. 